\title{
A CURIOSITY CONCERNING THE DEGREES OF THE CHARACTERS OF A FINITE GROUP
}

\author{
K. L. FIELDS
}

ABSTRACT. Let $G$ be a finite group with irreducible characters $\{\ldots$, $\chi, \ldots\}$ and $K=Q(\ldots, \chi, \ldots)$ the field generated over the rationals by their values. We will prove:

$$
\text { If } K=\mathbf{Q}(\text { or if }[K: \mathbf{Q}] \text { is odd }) \text { then } \prod_{\chi(1) \text { odd }} \chi(1) \text { is a perfect square. }
$$

More generally,

THEOREM. (a)

$$
(-1)^{(\Sigma \chi(1)-(m+1)) / 2} \prod_{\chi(1) \text { odd }} \chi(1)
$$

is a square in $K$ if $|G|$ is even.

(b) $(-1)^{(|G|-1) / 2}|G|$ is a square in $K$ if $|G|$ is odd.

$\left[\right.$ Recall $|G|=\Sigma \chi(1)^{2}$ so that $|G| \equiv \Sigma \chi(1) \bmod 2$, and so $\Sigma \chi(1) \equiv m+1$ mod 2 where $m$ denotes the number of involutions in $G$.]

Proof of Theorem. Let $F$ be any splitting field for $G$ such that char $F+G \mid$. Consider the $F$-vector space $F G$ on which we have the nondegenerate symmetric bilinear form defined by $B(g, h)=\rho(g h)$ for $g, h \in G$, where $\rho$ is the trace of the regular representation of $G$. So

$$
B(g, h)=\left\{\begin{array}{cc}
0, & g \neq h^{-1} \\
|G|, & g=h^{-1}
\end{array}\right.
$$

and with respect to this basis of group elements, $B$ is the direct sum of $(|G|-(m+1)) / 2$-matrices $\left(\begin{array}{c}0 \\ |G|\end{array} \mid \begin{array}{l}|G| \\ 0\end{array}\right)$ and $m+1(1 \times 1)$-matrices $(|G|)$. So the discriminant of $B$ is $(-1)^{(|G|-(m+1)) / 2}|G|^{|G|}$. But, if we identify $F G$ with the direct sum $\sum M_{\chi(1)}(F)$ of $(\chi(1) \times \chi(1))$ matrix algebras over $F$, and if $e_{i j}^{\chi}$ denote the matrix units, then

$$
B\left(e_{i j}^{\chi}, e_{k l}^{\lambda}\right)= \begin{cases}\chi(1), & i=l, j=k, \chi=\lambda, \\ 0, & \text { otherwise. }\end{cases}
$$

So with respect to this basis, $B$ is the direct sum of $\left(\chi(1)^{2}-\chi(1)\right) / 2$-matrices $\left(\begin{array}{cc}0 & \chi(1) \\ \chi(1) & 0\end{array}\right)$ and $\chi(1)(1 \times 1)$-matrices $(\chi(1))$, and so has discriminant

Received by the editors May 21, 1975 and, in revised form, October 21, 1975. AMS (MOS) subject classifications (1970). Primary $20 \mathrm{Cl} 5$. 


$$
\begin{gathered}
\prod_{\chi}(-1)^{\left(\chi(1)^{2}-\chi(1)\right) / 2}\left(\chi(1)^{2}\right)^{\left(\chi(1)^{2}-\chi(1)\right) / 2} \chi(1)^{\chi(1)} \\
=(-1)^{(|G|-\Sigma \chi(1)) / 2} \prod \chi(1)^{\left(\chi(1)^{2}\right)} .
\end{gathered}
$$

Hence

$$
(-1)^{(|G|-(m+1)) / 2}|G|^{|G|} \text { and }(-1)^{(|G|-\Sigma \chi(1)) / 2} \prod \chi(1)^{\left(\chi(1)^{2}\right)}
$$

differ by a square in $F$.

If $|G|$ is odd, then the nontrivial characters occur in conjugate pairs, so that II $\chi(1)^{\left(\chi^{\left.(1)^{2}\right)}\right.}$ is a square; moreover, since $\Sigma \chi(1)=1+2 \sum_{i=1}^{(c-1) / 2}\left(2 k_{i}+1\right)$ where $c$ denotes the number of conjugacy classes,

$$
\sum \chi(1) \equiv c \bmod 4 \text {. }
$$

Also, (Burnside) $|G| \equiv c \bmod 16$, so we have that $(-1)^{(|G|-1) / 2}|G|$ is a square in $F$.

If char $F=0$, then the intersection of all splitting fields $F$ is just $K$. [Given a simple algebra finite dimensional over a number field $L$, the GrunwaldWang and Tchebotarev Density Theorems imply the existence of a prime $p$ in $L$ and maximal subfields $F_{1}$ and $F_{2}$ such that $p$ splits completely in $F_{1}$ but is divisible by only one prime of $F_{2}$; hence $F_{1} \cap F_{2}=L$. An analogous result holds for a semisimple algebra all of whose simple components have the same center; in the case of a group algebra, this common center can be chosen to be $K$ above], and so our Theorem follows.

REMARKS. (1) If all the characters of $G$ are real, we must have

$$
(-1)^{(\Sigma \chi(1)-(m+1)) / 2}=1, \quad \text { i.e., } \sum \chi(1) \equiv m+1 \bmod 4 \text {. }
$$

(2) If $F$ can be chosen to be real, we have that

$$
\sum \frac{\chi(1)^{2}-\chi(1)}{2}=\frac{|G|-(m+1)}{2}=\text { Witt index of } B,
$$

and so $\Sigma \chi(1)=m+1$.

Both of these facts follow also from the classical Frobenius-Schur count of involutions.

(3) If $G$ is cyclic of odd order $n$, then Theorem (b) states that

$$
(-1)^{(n-1) / 4} \sqrt{n} \in \mathbf{Q}\left(\xi_{n}\right)
$$

(4) Recall that for even $|G|$, there are an odd number of nonprincipal, nonconjugate characters having both odd degree and an odd number of conjugates [Proc. Amer. Math. Soc. 30 (1971), 247-248]. In other words, $\Pi_{\chi(1) \text { odd }} \chi(1)$ is nontrivial, and is never square solely by virtue of conjugate characters.

(5) It would be of interest to find necessary and sufficient conditions on $G$ to insure that every character has an odd number of conjugates (this implies 
that the characters are real) i.e., that $[K: Q]$ is odd. It would also be of interest to determine exactly the discriminant of $K$. Also, it is unknown which abelian extensions of $\mathbf{Q}$ appear as $K=\mathbf{Q}(\ldots, \chi, \ldots)$ for some finite group G.

(6) We thank F. Gross and G. Walls for simplifying our original statement for odd $|G|$.

Department of Mathematics, Rider College, Lawrenceville, New Jersey 08648 